\title{
The PLATO mission
}

\author{
Heike Rauer ${ }^{1,2}$, Claude Catala ${ }^{3}$ and the PLATO consortium \\ ${ }^{1}$ Institut für Planetenforschung, DLR, \\ Rutherfordstr. 2, 12489 Berlin, Germany \\ email: heike.rauer@dlr.de \\ ${ }^{2}$ Zentrum für Astronomie und Astrophysik, TU Berlin \\ 10623 Berlin, Germany \\ ${ }^{3}$ LESIA, Observatoire de Paris \\ 5 Place Jules Janssen, 92195 Meudon Cedex, France \\ email: claude.catala@obspm.fr
}

\begin{abstract}
The PLAnetary Transits and Oscillations of stars (PLATO) mission is in its definition study phase in the context of ESA's Cosmic Vision 2015-2025 program. PLATO is applying for a launch in $2017 / 18$. Its goal is to detect transiting exoplanets, including terrestrial planets in the habitable zone, and to determine their basic parameters with unprecedented accuracy. In combination with the detailed analysis of the stellar parameters by astroseismology and with ground-based follow-up observations, this will allow characterizing the main properties of exoplanetary systems to a level not achieved before.
\end{abstract}

Keywords. instrumentation: photometers, techniques: photometric, stars: evolution, stars: fundamental parameters, planetary systems

\section{Introduction}

The PLAnetary Transits and Oscillations of stars (PLATO) mission is the next generation space mission to detect transiting extrasolar planets in the solar neighbourhood. PLATO follows the successful CoRoT (CNES) and Kepler (NASA) missions, but aims to detect a sample of planetary systems with terrestrial planets orbiting well-characterized bright stars, including planets in their habitable zone. This new sample of planetary systems will have accurately known basic planet parameters (radius, mass, orbit, age). Planet radii and masses will be derived from highly accurate photometric transit observations with PLATO, combined with a world-wide ground-based follow-up campaign including radial velocity follow-up measurements. Stellar parameters across the HR diagram and for central stars of planetary systems are studied by astroseismology using PLATO's well time-sampled and highly accurate photometric lightcurves. The resulting large sample of such well-known planets orbiting bright stars will provide a breakthrough for numerous further scientific studies.

Examples of the expected scientific impact of PLATO in the field of exoplanets include the study of the planet interior via mass-radius relationships, the orbital evolution of planetary systems, and atmospheric spectral characterization with future spectroscopic telescopes. Furthermore, PLATO has the potential to provide input for new insights into planet evolution. Today, a detailed study of planetary evolution as a fucntion of the age of the system can be made only for the Solar System. We have no information as to whether planets in other planetary systems develop similarly to the well-known planets around the Sun. Therefore, placing our Solar System into a wider context of planetary evolution is currently not feasible with sufficient accuracy because the accuracy of known 
Table 1. Number of cool dwarf stars and subgiants in PLATO fields. Cols. 2 and 3: long-term monitoring phase; col. 4: including step-and-stare phase; cols. 5 and 6: comparison with KEPLER; given as a function of noise level (top) and of magnitude (bottom)

\begin{tabular}{|l|l|l|l|l|l|}
\hline & PLATO $\left(4300 \mathrm{deg}^{2}\right)$ & $\mid 20,000 \mathrm{deg}^{2}$ & \multicolumn{2}{|c|}{ KEPLER $\left(100 \mathrm{deg}^{2}\right)$} \\
\hline noise level (ppm/sqrthr) & nb of stars & $\mathrm{m}_{v}$ & nb of stars & nb of dwarf stars & $\mathrm{m}_{v} \mid$ \\
\hline 27 & 20,150 & $9.3-10.8$ & 80,400 & 1,300 & 11.2 \\
80 & 292,000 & $11.6-12.9$ & $1,000,000$ & 25,000 & 13.6 \\
\hline & 1,326 & 8 & 3,315 & 30 & $\mid \begin{array}{l}8 \\
11\end{array}$ \\
\hline
\end{tabular}

ages of extrasolar planets is limited by the only poorly constrained ages of main sequence stars.

PLATO addresses this need for significantly improved stellar parameters, including their ages, by the combination of exoplanet transit detections with astroseismology of their central stars. The accuratly known radii and masses will put meaningful constraints on mass-radius relationships and planet interior. In addition, it has been shown (Kjeldsen et al. 2009) that astroseismology with PLATO allows us to derive stellar ages to within $10 \%$. PLATO is therefore expected to provide the basis for a wealth of new discoveries in our understanding of planet and stellar evolution.

PLATO one out of three space missions which are applying for the M2 launch window for medium-sized missions in 2017/18 in the context of ESA's Cosmic Vision program (Stankov et al. 2010). At the time of writing, PLATO is in the Definition Phase, in which the spacecraft design will be consolidated and optimized.

\section{Mission goals}

The prime science goals of PLATO are (Catala et al. 2009; Claudi et al. 2009): i) The detection and characterization of Earth Analog systems. ii) The search for exoplanets around the brightest stars of solar type at all orbital periods and with all physical sizes. iii) Search for exoplanets around nearby M-type dwarfs with all physical sizes and at all orbital periods, including at orbital distances such that these planets fall within the habitable zones of these very cool stars. iv) Search for and characterization of exoplanets with a wide variety of sizes, masses and orbits around bright stars. v) Full characterization of very bright stars, of all masses and ages, using seismic analysis.

The PLATO mission will detect exoplanets by the transit method and measure the seismic oscillations of the parent stars in order to fully characterize the basic parameters of exoplanetary systems. These goals will be achieved by a long-term, ultra-high precision monitoring in visible photometry of bright dwarfs and subgiants, as well as of a large sample of M dwarfs. The major breakthrough will come from PLATO's strong focus on bright targets, typically with $\mathrm{m}_{V} \leqslant 11 \mathrm{mag}$. The PLATO targets will also include a large number of very bright and nearby stars, with $\mathrm{m}_{V} \leqslant 8 \mathrm{mag}$, as well as a large sample of cool $\mathrm{M}$ dwarfs down to $\mathrm{m}_{V}=15-16$ mag.

The PLATO observations will be complemented by a world-wide effort of ground-based follow-up observations, including radial velocity monitoring. In comparison to ongoing space missions for exoplanet detection, spectroscopic follow-up observations to confirm the planet nature of a transit event and to determine the planet mass will be significantly facilitated by the brightness of the PLATO targets. 


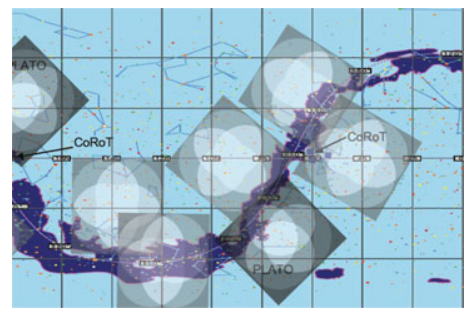

Figure 1. Illustration of sky coverage. Exact field positions will be defined during study phase.

\section{The target samples}

The prime focus of PLATO is on planets orbiting bright stars. The highest priority target samples, therefore, are about 20,000 dwarfs and sub-giants with $\mathrm{mV} \leqslant 11$ mag. This sample will be monitored with a noise level better than $27 \mathrm{ppm}$ in one hour. This noise level is sufficient to detect Earth-sized exoplanets and to well characterize the central stars by astroseismology. In addition, a sample of at least 1,000 very bright dwarfs/subgiants with $\mathrm{mV} \leqslant 8 \mathrm{mag}$ will be observed. This sample of very bright stars will provide the prime targets for further spectral characterization of the detected planets, e.g. for atmosphere studies, by spectroscopic telescopes. Finally, more than 245,000 cool dwarfs/subgiants will be observed at a relaxed noise-level (better than $80 \mathrm{ppm}$ in one hr). This sample will still allow detecting transit signals from terrestrial planets around solar-type stars, but radial-velocity confirmation and astroseismology will be difficult due to the faintness of the stars (about $11 \mathrm{mag}<\mathrm{V}<13 \mathrm{mag}$ ). Nevertheless, this sample will provide a large number of planets covering a wide range of parameter space. The expected approximate number of target stars in the PLATO fields is given in Table 1. To increase the detected number of planets close to the habitable zone of their central star with PLATO, a target sample of more than 10,000 M dwarfs has been added. This target sample will be particularly interesting for further spectroscopic characterizing observations.

It is planned to launch PLATO into an orbit around the Sun-Earth L2 Lagrange point to allow for long-duration observations. Two prime target fields will be monitored for at least 2 - 3 years with high duty cycle, typically above $95 \%$, and high time sampling. In addition, several fields of shorter duration (2-5 months each) will be monitored ("stepand-star" phase) for 2-3 years. In total, about $50 \%$ of the sky will be covered at the end of the mission. The sky coverage is illustrated in Fig. 1. However, the final selection of the exact target field positions will be made later during the PLATO study phases.

\section{The instrument concept}

The instrument concept of PLATO differs from traditional space observatories (Catala et al. 2009). As a result of the required large number of target stars over a wide magnitude range, a multi-telescope approach was chosen (Fig. 2, left). Observations of 32 refractive telescopes are combined to produce highly photometrically accurate lightcurves. Each telescope has a pupil size of about $120 \mathrm{~mm}$ and operates in "white" light. They have a read-out cadence of $25 \mathrm{sec}$, which is, however, binned later to increase the signal-tonoise ratio (SNR) depending on star magnitude (50 sec for the highest priority bright star samples, and better than $600 \mathrm{sec}$ for the fainter star samples). The telescopes are complemented by two so-called "fast" telescopes with higher read-out cadence $(2.5 \mathrm{sec})$ and fixed colour filters. These two telescopes are used to monitor the brightest targets, 
Table 2. Instrument characteristics.

\begin{tabular}{|l|l|}
\hline pupil diameter per telescope & $120 \mathrm{~mm}$ \\
normal telescope field-of-view & $\sim 1100 \mathrm{deg}^{2}$ (38.7 diameter) \\
normal telescope detector & $4510^{2}$ pixels, $18 \mu \mathrm{m}$ square \\
fast telescope field-of-view & $\sim 5500 \mathrm{deg}^{2}$ \\
fast telescope detector & $4510 \mathrm{x} 2255$ pixels, $18 \mu \mathrm{m}$ square \\
plate scale & 15 arcsec/pixel \\
total field-of-view (FoV) & overlapping FoV of $2232 \mathrm{deg}^{2}$ or $42.4 \times 42.4 \mathrm{deg}^{2}$ \\
\hline
\end{tabular}
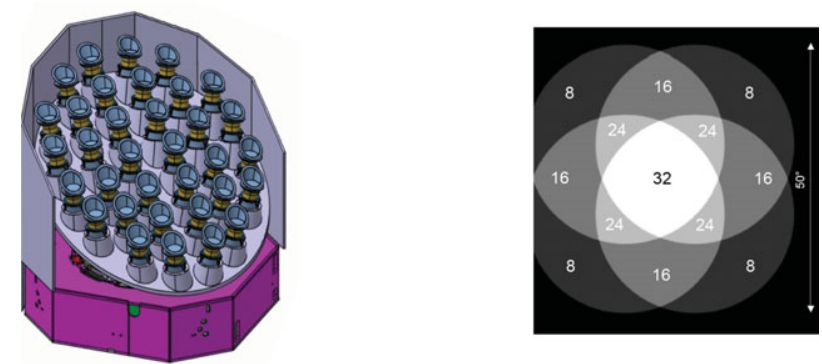

Figure 2. Instrument concept (left) and overlapping viewing directions (right).

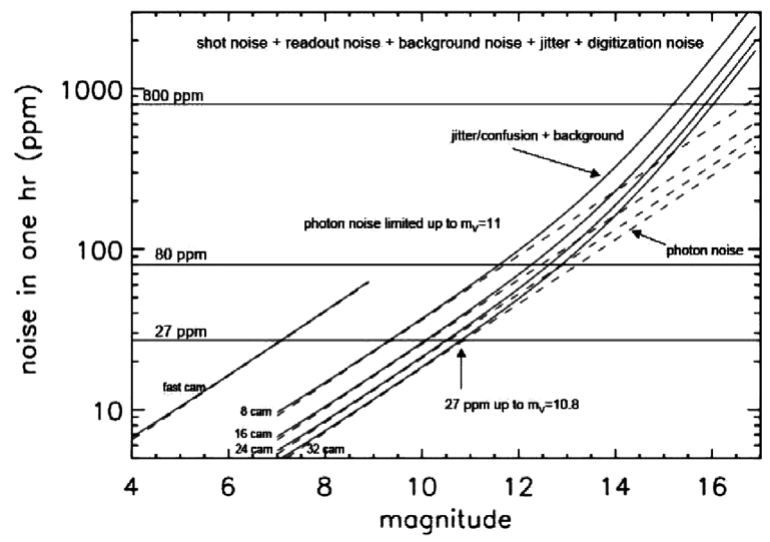

Figure 3. Instrument performance.

but also for satellite fine-pointing. The main foreseen instrument characteristics are given in Table 2.

The 32 "normal" telescopes are combined into 4 groups of 8 telescopes each, pointing into the same viewing direction. The fields of the 4 groups overlap, thereby increasing the area covered in one pointing but keeping a central area where all 32 telescopes are combined (Fig. 2, right). This setting is an optimization of the number of stars at a given noise level and the number of stars at a given magnitude range. Fig. 3 shows the expected noise level for different combinations of telescopes. We note that it is in principle possible to come back to a previous pointing during the "step-and-stare" phase to re-observe interesting targets.

\section{Summary}

PLATO is the next logical step in the investigation of extrasolar planets. The presently known transiting extrasolar planets have demonstrated the wealth of information that can be gained for these objects, e.g. on their internal structure from their mass and radius, their dynamical history from their orbital parameters (including Rossiter-McLaughlin 
effect), their atmospheric composition from spectroscopic follow-up. However, the presently known transiting extrasolar planets have also shown where our limitations are to derive conclusive interpretations of what we see, on the observational side as well as on our understanding of planetary systems. Today, the accuracy of mass and radius is limited by our knowledge of the central star. This is in particular true for the most interesting objects: the small, terrestrial planets. Furthermore, up to now a real evolutionary comparison of planets can not be made due to the poorly constraint stellar ages. Since atmospheres can only be detected for a limited number of planets around very bright stars, such investigations are strongly limited so far. All of these current limitations are addressed by the PLATO mission by combining the detection of transiting planets with the detailed analysis of their central stars and stellar evolution in general by astroseismology. In addition, the target sample of PLATO focusses on bright stars which will significantly improve our abilities to investigate these objects in more detail in future. Thus, PLATO will lay the ground for a breakthrough in our understanding of planetary as well as stellar evolution.

\section{References}

Catala, C. 2009, Communications in Asteroseismology, 158, 330

\&Claudi, R., et al. 2009, APESSS, 218, 319

Kjeldsen, H., Bedding, T. R., \& Christensen-Dalsgaard, J. 2009, in Transiting Planets, Proc. IAU Symp., 253, 309

Stankov, A., Baldesarra, M., Piersanti, O., Fridlund, M., Lindberg, R., \& Rando, N. 2010, in Society of Photo-Optical Instrumentation Engineers, Proc. SPIE, 7731, 45 